\title{
Role of Doppler Ultrasonography Supplemented with Direct CT Venography in Dialysis Access Assessment
}

\author{
Ahmed F. Yousef ${ }^{\mathrm{a}}$, Hamada M. Khater ${ }^{\mathrm{a}}$, Hend A. AbdElrazik ${ }^{\mathrm{a}}$
}

\footnotetext{
Department of radiology, Benha faculty of medicine, Benha university, Egypt correspondence to: Hend A. AbdElrazik (MSC), Department of radiology, Benha faculty of medicine, Benha university, Egypt

email: r.elbahr@yahoo.com
}

Received:27 October 2019

Accepted: 3 November 2019

BMJ 2019, vol 36, issue 2

\section{Background}

Functioning dialysis vascular access is essential for end stage kidney disease patients

\section{Purpose}

To review the value of following a systematic DUS protocol of examination in DVA malfunction assessment. Through following this protocol, we will review the role of d-MDCTV examination in completion of DVA assessment in selected cases in which DUS assessment is incomplete.

\section{Patients and methods}

We evaluated 50 patients with malfunctioning DVA. The study population had been categorized into 2 groups; Group A (25 patients): was examined by DUS only, Group B (25 patients): was examined by DUS followed by d-MDCTV, using 8 channels CT scanner, with injection of 130-150 ml diluted contrast media into any accessible vein in the limb harboring dialysis vascular access

\section{Results}

Regarding both studied groups; different pathologies were diagnosed to cause DVA malfunction, (anastomosis/ venous thrombosis(17), anastomosis stenosis(5), peripheral venous stenosis(9), deep position of the VA(2), venous aneurysm/ pseudoaneurysm(8), branchouts(11), venous hypertension(5), arterial thrombosis(4), arterial stenosis(3), arterial aneurysm (4)\& Dialysis induced limb ischemia(8). Diagnosis of these pathologies were almost well established by DUS alone As 
regarding the evaluation of central veins for group B patients; 18 patients were confirmed by d-MDCTV to have a central venous occlusive disease; the diagnosis in 4 of them was equivocal by DUS. 7 patients were confirmed by d-MDCTV to have a normal central veins; the diagnosis in 1 of them was equivocal by DUS.

\section{Conclusion}

A systematic DUS examination protocol is the 1st line in DVA malfunction assessment. This assessment in certain cases is incomplete. We suggest that d-MDCTV can complete this assessment.

\section{Key words:}

\section{d-MDCTV; DUS; CVOD}

\section{Introduction}

Functioning DVA is essential for ESRD patients. AVFs and AVGs, the preferred VA for dialysis, are problematic; AVFs have a high rate of maturation failure and AVGs have a short life expectancy (1)."Vascular Access Clinical Practice Guidelines of the ESVS" have been published in 2018. It included pivotal roles for diagnostic radiology in DVA surveillance \& malfunction assessment In patients with AVFs, DUS can assess fistula maturity \& a treatable cause of the persistent immaturity can often be identified. Furthermore, DUS can also demonstrate causes of fistula dysfunction, such as stenosis, in poorly functioning fistulas allowing for treatment prior to total fistula loss In patients with AVGs dysfunction, DUS can identify AVG thrombosis, the most common cause of failure. More importantly, DUS can identify vascular stenosis prior to thrombosis, allowing for potential treatment and preservation of the AVG (1). The introduction of a well-defined standardized
DUS protocol based on anatomic and hemodynamic parameters facilitates the multidisciplinary management of DVA, its monitoring and the timely prevention of any complications (2).

Nevertheless, DUS has its well-known drawbacks:The diagnostic quality of DUS depends strongly on the experience of the examiner.

1- It provides no angiographic map for the guidance of further therapy.

2- High inter-reader variability.

3- Limited evaluation of the central veins because of its deep location behind thoracic bones.

If CVOD cannot be reliably excluded by DUS, additional imaging methods will be necessary (3).

MSCTV is the first choice to evaluate the condition of CVODs in hemodialysis patients. It can provide accurate evaluations of the conditions of occlusive lesions, which can be of great clinical significance to the subsequent interventional therapy (4). Designing vascular imaging CT protocols can be challenging owing to the non-uniform velocity of blood, differences in cardiac output between patients, and the effect of the different pathological states on blood flow that cannot be predicted pre-scan (5).

\section{In addition, MDCTV has several drawbacks:}

Use of intravenous (IV) contrast media (CM): In patients with ESKD the plasma CM concentration remains high for a long period of time. Such patients are at the risk of central nervous system reactions such as convulsions and respiratory depression (6). Nevertheless, The Safety Committee (CMSC) concluded that there is no benefit to schedule the 
injection of the $\mathrm{CM}$ in relation to the dialysis program (6).

1- Radiation exposure.

2- It is not possible to perform a direct corrective intervention using this study.

So; combined use of DUS \& MDCT will significantly raise the success rate in detection of nearly all VA lesions, to be nearly equal to the diagnostic value of surgery as a gold standard (6). Accordingly, we tried, in our research, to follow \& review these guidelines in commensurate with our available resources.

\section{Purpose}

The aim of this prospective study is to review the value of following a unified systematic DUS protocol of examination in DVA malfunction assessment \& data prescription. Through following this protocol, we review the role of direct MDCTV study in completion of DVA assessment in selected cases in which the DUS assessment is incomplete.

\section{Patients and methods:}

\section{Patients:}

This study was prospectively carried on 50 patients, 31 females (62\%) and 19 males (38\%), with malfunctioning DVA. Patients were renrolled from Benha teaching hospital center of Nephrology and Dialysis, within a period of 20 months, to our radiology department in Benha teaching hospital from January 2018 up to September 2019, after approval of the ethical committee of Benha Faculty of Medicine \& after obtaining an informed consent from all the patients.

\section{Methods:}

We categorized study populations into 2 groups:

Group A: 25 patents (50\%) had been evaluated by DUS examination only.

Group B: 25 patents (50\%) had been evaluated by DUS study followed by dMDCTV, using 8 channels CT scanner (HITACHI). 28 upper limb d-MDCTV examinations had been performed for 25 patients; 22 unilateral \& 3 bilateral.

Inclusion criteria for DUS: patient having malfunctioning DVA and a decision is needed about surveillance of the current VA versus construction of a new one and about determination of the most suitable site for new VA construction.

Exclusion criteria for DUS: none.

DUS Protocol: B-mode in transverse \& longitudinal planes, together with color \& spectral Doppler facilities had been used. We followed a unified systematic protocol of examination in which:

1. We started by central veins examination, via studying the anatomy\& wave form pattern of the subclavian vein $(\mathrm{SCV}) \&$ internal jugular vein (IJV), and comparing them with the contralateral side. If the findings were equivocal, we recommended in the report further $\mathrm{d}$ MDCTV examination to complete the evaluation.

2. Afterwards, we examined the arterial inflow, anastomosis \& venous access regarding, their anatomy, peak systolic velocity (PSV), flow direction, as well as flow volume (FV).

3. As regarding to $A V G$, we studded the 2 anastomotic points as well as the cannulation points searching for stenosis, 
4. in addition to measuring the FV within the graft.

5. If the patient suffered from limb ischemia, then examination of the arterial outflow distal to the fistula/ graft and studing its flow direction and presence of any stenosis would be manadatory. If the flow was retrograde or very week, we temporary compress the DVA \& re-investigate the flow.

6. The FV had been measured mainly along the brachial artery. Also, it was measured in the arterial inflow as well as DVA if uniform.

7. Illustrative diagrams had been drawn to facilitate the case demonstration for the clinician (case 1: figures 1-6).

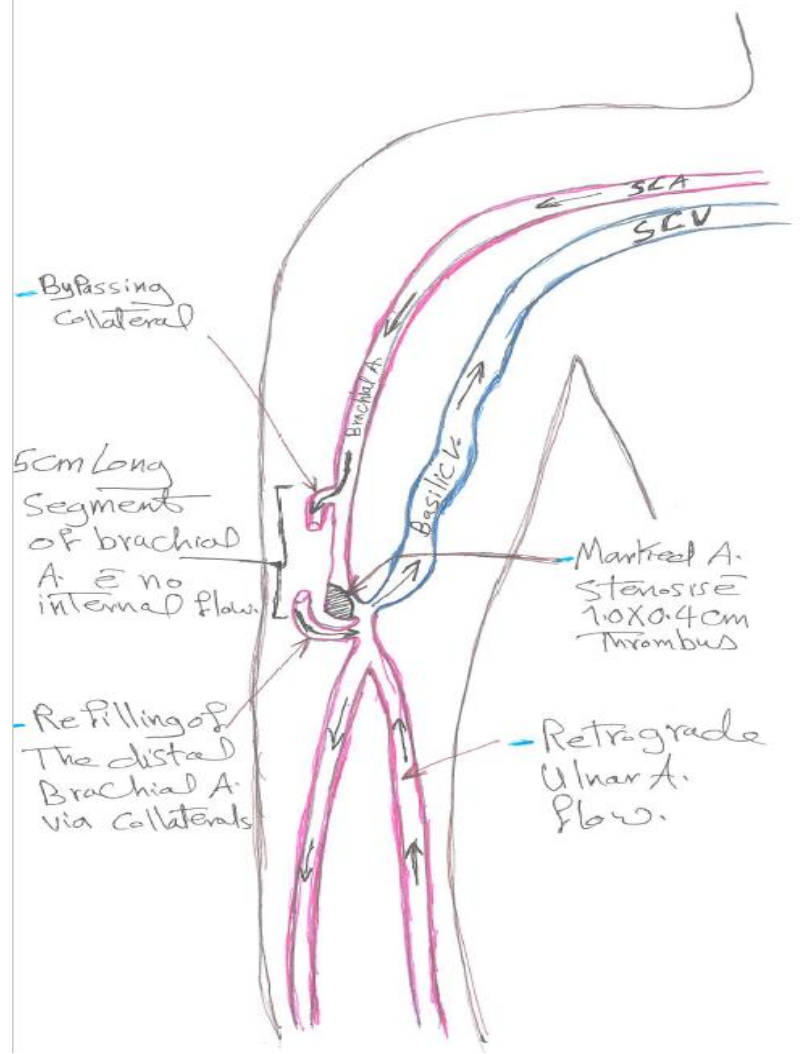

Fig.(1): Illustrative diagram for the case 1

\section{Inclusion criteria for d-MDCTV: If CVOD} was suspected or cannot definitely been excluded, or if the clinician asked for confirmatory data about the central veins. Exclusion criteria for d-MDCTV: COVD had been excluded confidently by DUS and there was no clinical suspicion for it.

d-MDCT Protocol: A diluted 130-150 ml low osmolar, non-ionized CM $(50 \mathrm{ml} \mathrm{CM} \mathrm{:}$ 80-100 saline) in order to decrease possible drawbacks of CM upon patient's organs. The site of injection was any accessible vein in the dorsum of the hand or forearm of the limb harboring DVA. The imaged arm was positioned beside the patient's trunk. Caudocranial 1st pass acquisition started at cannula level up to the thoracic inlet. None of the group B patients had experienced an allergic reaction or study induced significant vascular injury.

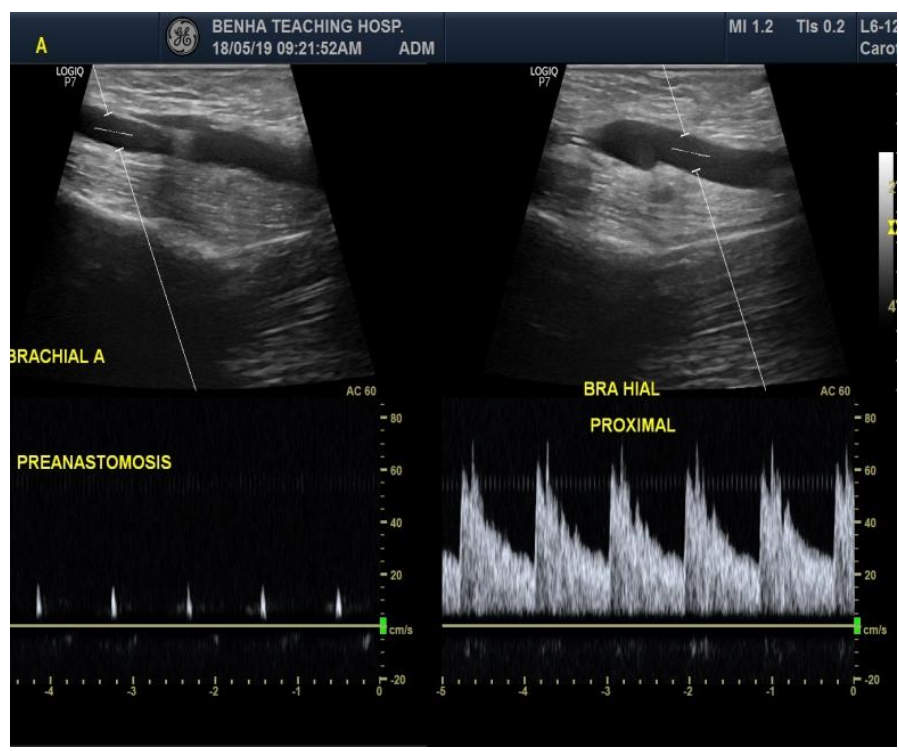

Figure 2- (Case 1): Female patient with malfunctioning brachio-basilic AVF: A) Spectral Doppler showing: Very week flow within the mid portion of the brachial artery (left) compared to normal flow within its proximal portion (right). 


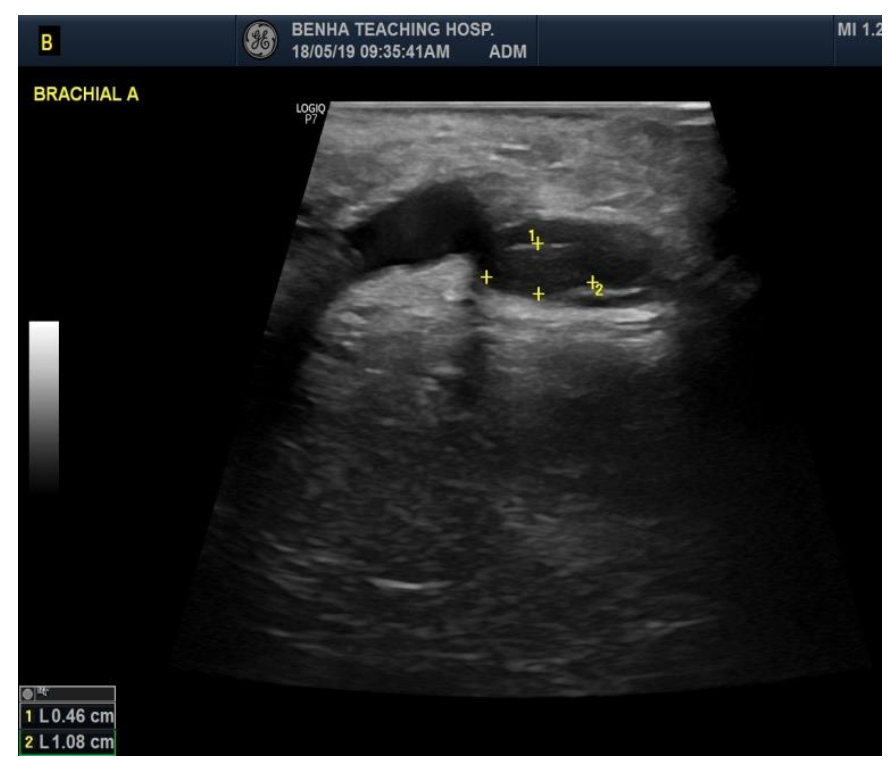

Figure 3- (Case 1): B) B- mode: Small thrombus within the distal portion of the brachial artery together with stenosis (Arrow) at its distal/ pre-anastomotic portion.

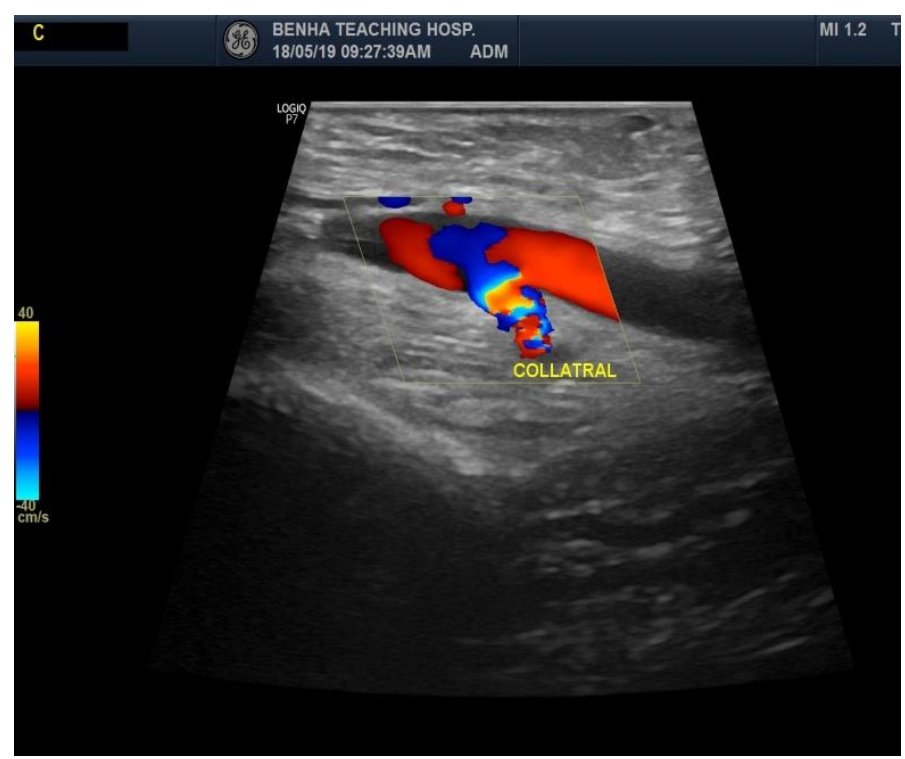

Figure 4- (Case 1) :C) Color Doppler: Bypassing collateral diverting blood flow from the mid brachial

A. segment to its distal / pre-anastomotic segment.

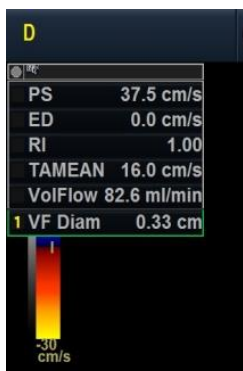

UA MID FOR ARM

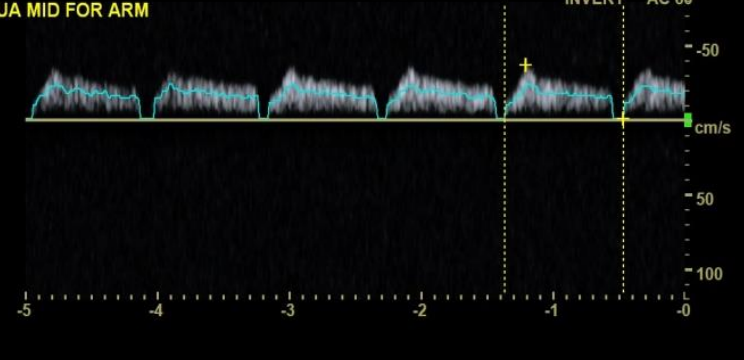

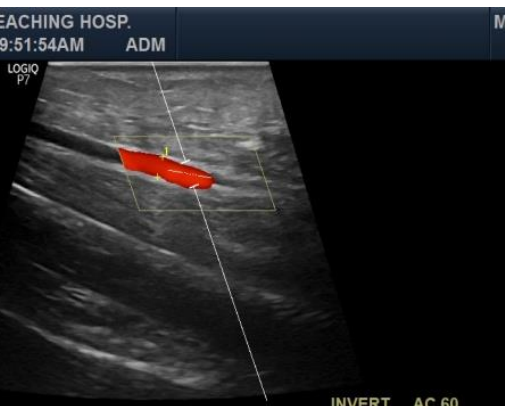

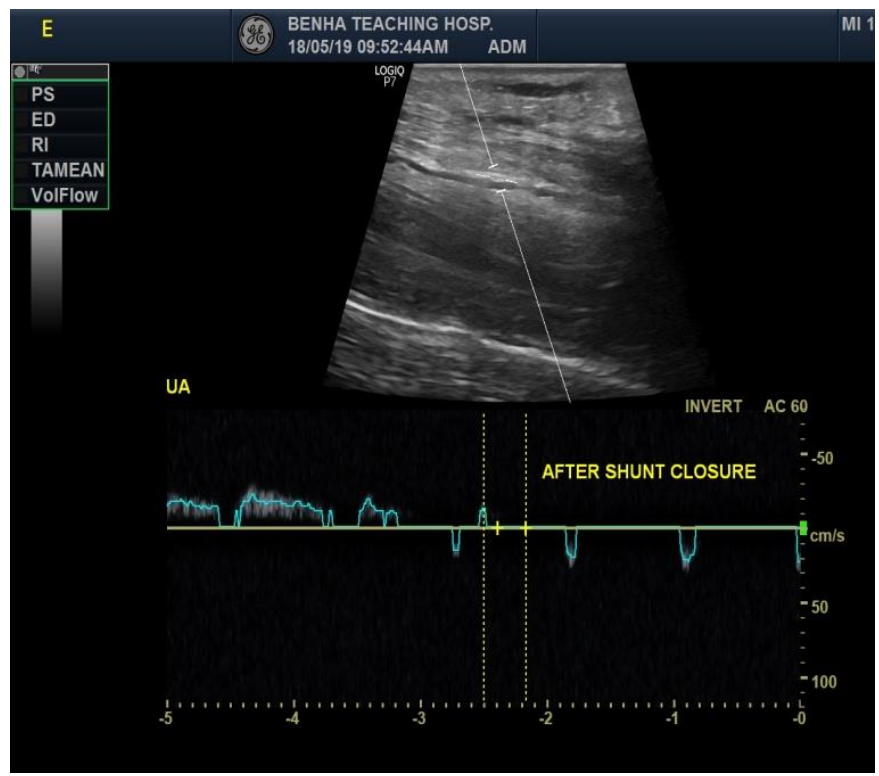

Figure 6- (Case 1): E) Normalization of the flow direction of the ulnar artery after temporary compression of the dialysis access.
Figure 5-(Case 1):D) Abnormal wave form of the ulnar artery suggesting steal. 


\section{Results}

Dialysis Access type: 45 patients $(90 \%)$ have had upper limb AVF, 4 patients (8\%) had AVG \& 1 patient $(2 \%)$ had axillary arterio-arterial graft.The overall pathologies demonstrated in both A \& B study groups are illustrated in (fig 7).

As regarding both A \& B groups patients; different pathologies had been diagnosed as causes of their DVA malfunction, as follows; anastomosis/ venous side thrombosis (17 patients / $34 \%$ ), anastomosis stenosis (5 patients $/ 10 \%$ ), peripheral venous access/ drainage stenosis (9 patients /18\%), deep position of the VA (2 patients $/ 4 \%$ ), venous aneurysm/ pseudoaneurysm (8 patients $/ 16 \%$ ), branchouts (11 patients $/ 22 \%$ ), venous hypertension (5 patients/10\%), arterial thrombosis (4 patients $/ 8 \%$ ), arterial stenosis (3 patients $/ 6 \%$ ), arterial aneurysm (4 patients $/ 8 \%$ ) \& Dialysis induced limb ischemia/ high flow fistula (8 patients $/ 16 \%$ ). Diagnosis of all those pathologies had been almost well established by DUS examination alone. d-MDCTV added in the confirmation of some of them and failed to demonstrate others.

As regarding group A (25 patients) study sample: the cause of DVA malfunction had been reached \& well demonstrated by the systematic DUS protocol with no need for further diagnostic imaging modality use. Surgical intervention was performed for 15 patients \& had confirmed our diagnostic data, e.g. banding the high flow fistula, ligation of branchouts \& superficialization of deeply seated access (fig 8). 10 cases managed conservatively.

As regarding the evaluation of central veins for group B (25 patients) (fig 9): 18 patients (72\%) had been confirmed by d-MDCTV to have (CVOD); the diagnosis in 4 patients (16\%) of them was equivocal during evaluation by DUS (Case 2: figures 10-13). 7 patients (28\%) had been confirmed by d-MDCTV to have normal central veins; the diagnosis in one patient $(4 \%)$ of them was equivocal during evaluation by DUS, due to nonspecific SCV wave form pattern was nonspecific because its pattern was composite of the transmitted arterial pulsation (through the VA) and the right atrial pulsatility. As regarding to other causes of DVA malfunction than CVODs in group B study sample (25 patients), the diagnosis had been almost well established by DUS. dMDCTV added in the confirmation of some of them and failed to demonstrate others, (fig 14), as follows:

- Nine patients have had venous thrombosis. 7 of them had been demonstrated well by each DUS \& d-MDCTV studies; however, d-MDCTV failed to demonstrate 2 of them.

- Seven patients have had sizeable branch-outs within $10 \mathrm{~cm}$ from the anastomosis, 5 of them had been demonstrated well by each DUS\& dMDCTV studies; however, d-MDCTV failed to adequately demonstrate 2 of them. Raw data 2D images were better than $3 \mathrm{D}$ images in the picking up of those branchouts.

- Four patients have had venous hypertension that could be diagnosed by DUS study and had been confirmed by d-MDCTV (Case 2 ).

- Three patients have had partially thrombosed venous aneurysms or pseudo-aneurysms and 3 patients have had venous access stenosis. All of them had been demonstrated successfully by each studies.

- Three patients have had anastomosis stenosis demonstrated only by DUS study, but dMDCTV failed to demonstrate any of them.

- One patient has had an Axillary artery partially thrombosed aneurysm followed by brachial artery total thrombosis diagnosed by DUS study. d-MDCTV succeeded in demonstration of the aneurysm and failed in demonstration of the arterial occlusion (Case 3: figures 15-18) 
Considering surgery as a gold standard, which was performed for $80 \%$ of our patients, our diagnostic data were highly matched with surgical findings (fig 8).

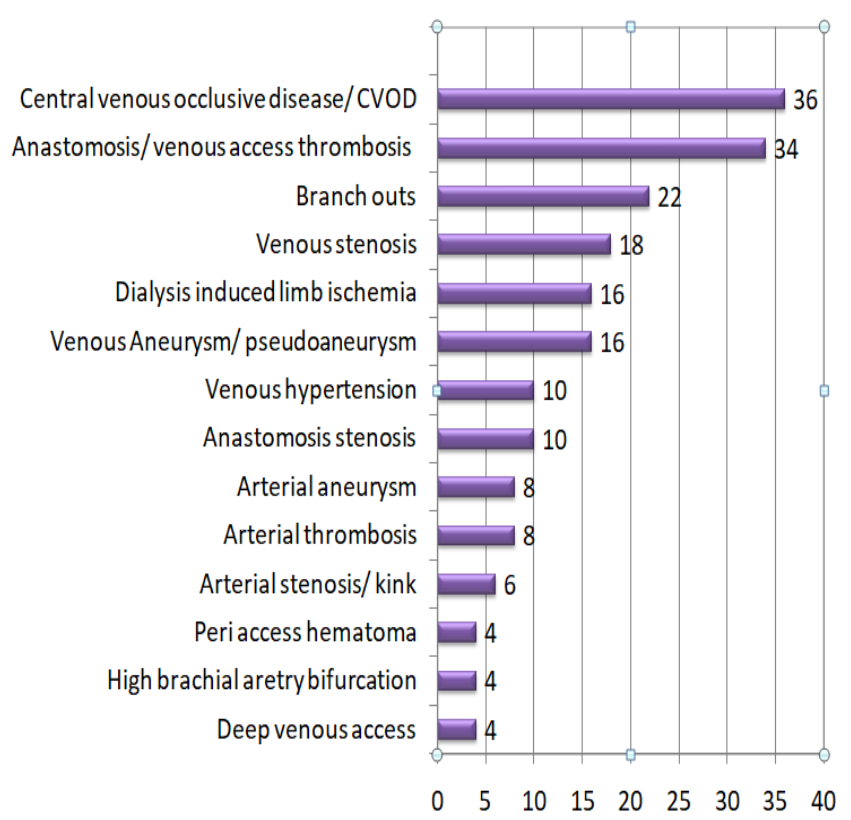

Figure 7- Bar chart representing the percentile of different pathologies detected as a cause of DVA.

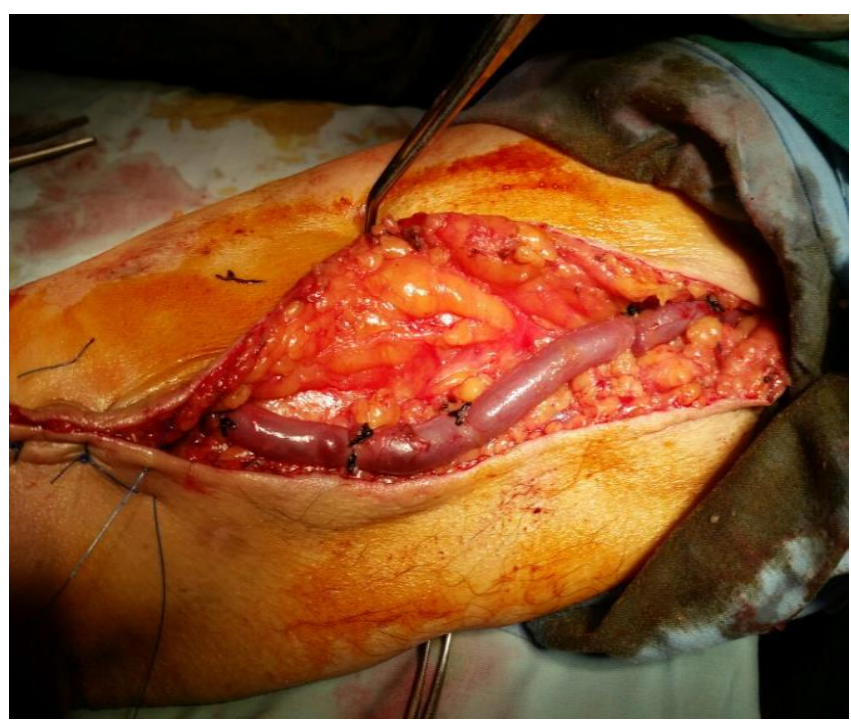

Figure 8- Intra operative image showing superficialization of DVA after its evaluation by DUS which was reported as "the cause of DVA" malfunction is its deep position".

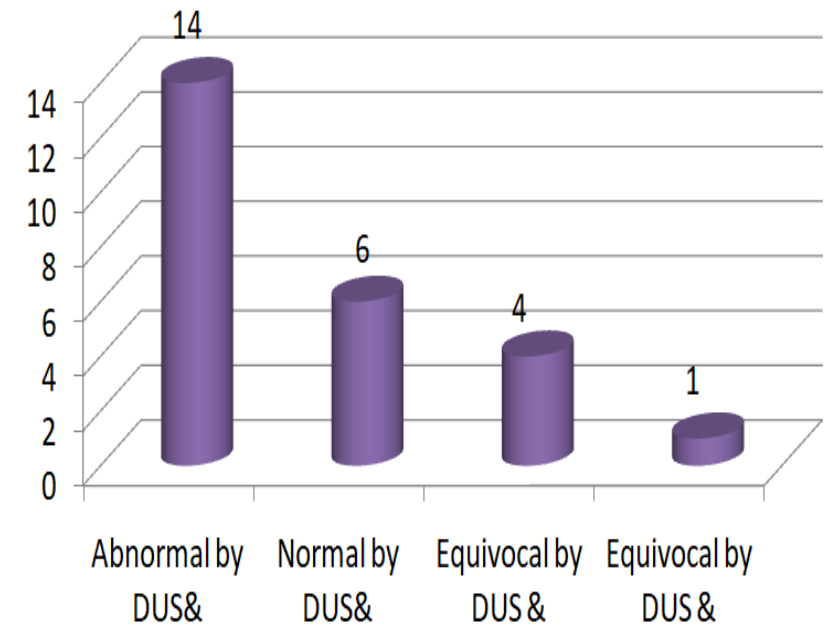

Abnormal by Normal by d- Abnormal by Normal by dd-MDCTV MDCTV d-MDCTV MDCTV

Figure 9- Bar chart representing the value of DUS study versus d-MDCTV in the evaluation of central veins for group B study sample patients

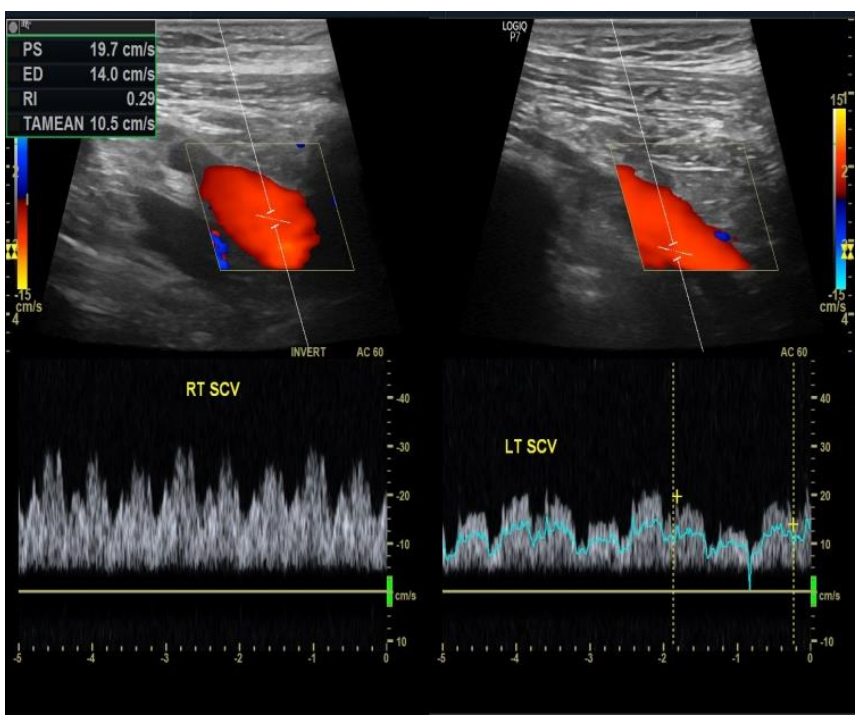

Figure 10- (Case 2): Female patient with malfunctioning right brachio-basilic AVF: A) Spectral Doppler images showing equivocal wave form pattern perceived from right Subclavian vein compared to the wave from pattern of the left SCV. 


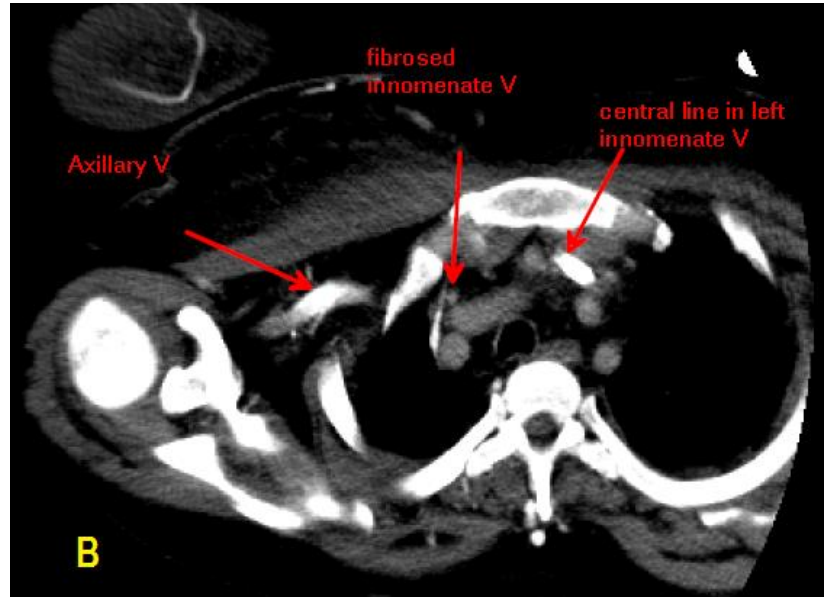

Figure 11- (Case 2): B) Axial d-MDCTV image showing fibrosed right innomenate vein with central catheter for dialysis seen on the left side.

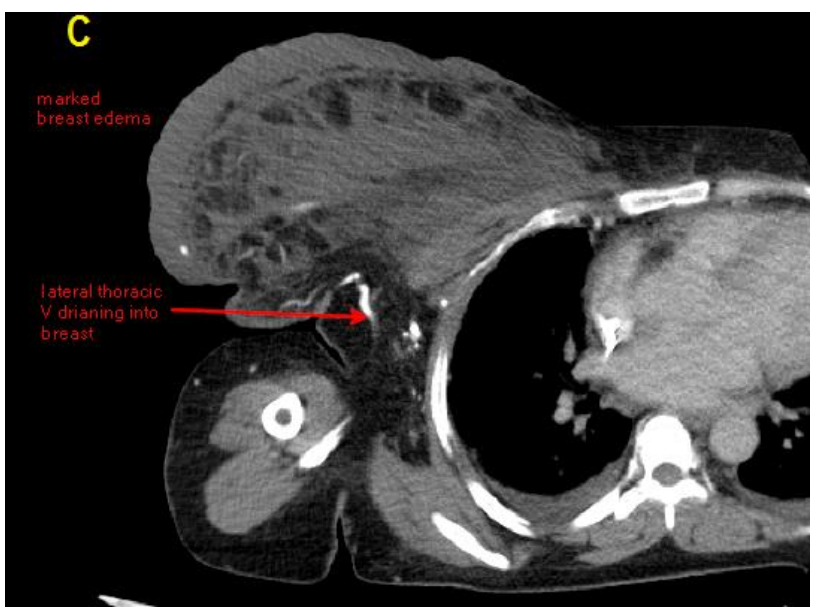

Figure 12- (Case 2): C) Axial d-MDCTV image showing: Marked right breast edema due to venous hypertension as a consequence of abnormal drainage of the right brachial vein into the lateral thoracic vein

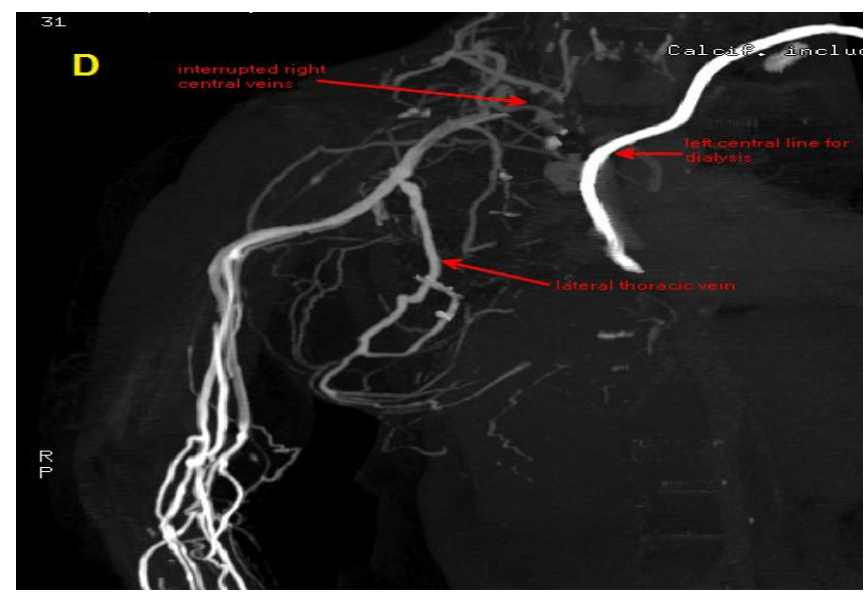

Figure 13- (Case 2): D) d-MDCTV - 3D image illustrating the fore mentioned findings.

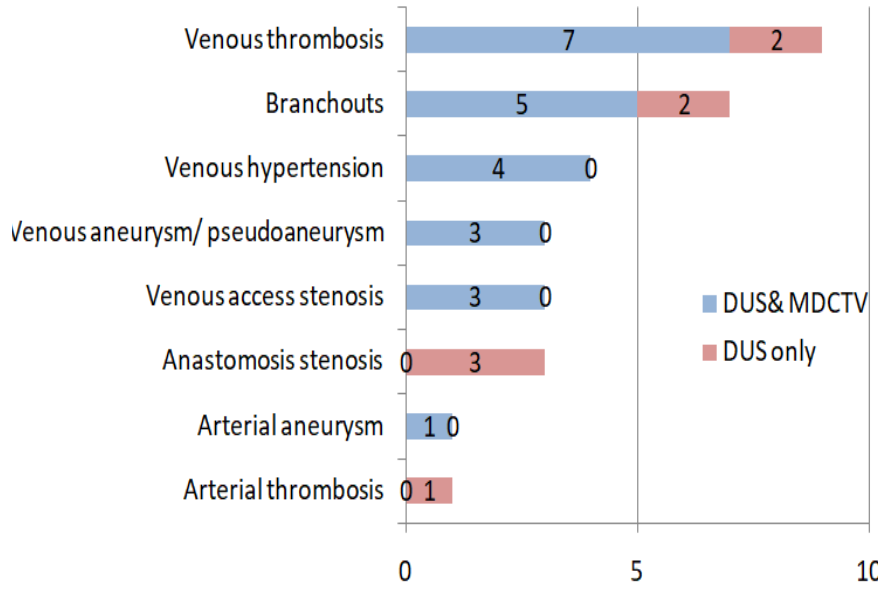

Fig 14 Chart representing number of Group B patients whom causes of DVA malfunction, other than CVOD, had been demonstrated by d-MDCTV (blue), compared to patients whom the causes couldn't be demonstrated (red) by it

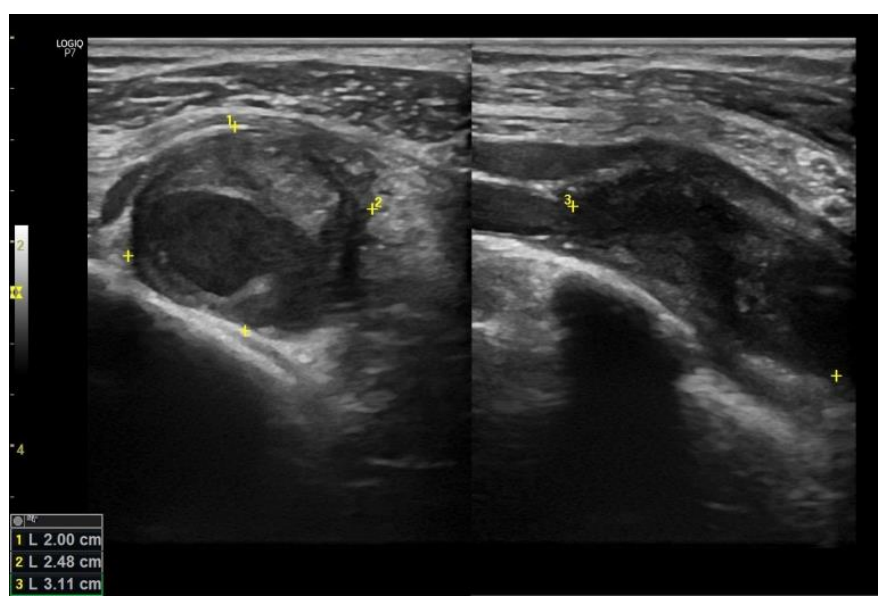

Figure 15- (Case 3): Male patient with acute on top of chronic malfunction of left side radiocephalic AVF: A) DUS, B-mode axial\& longitudinal views of axillary artery partially thrombosed aneurysm.

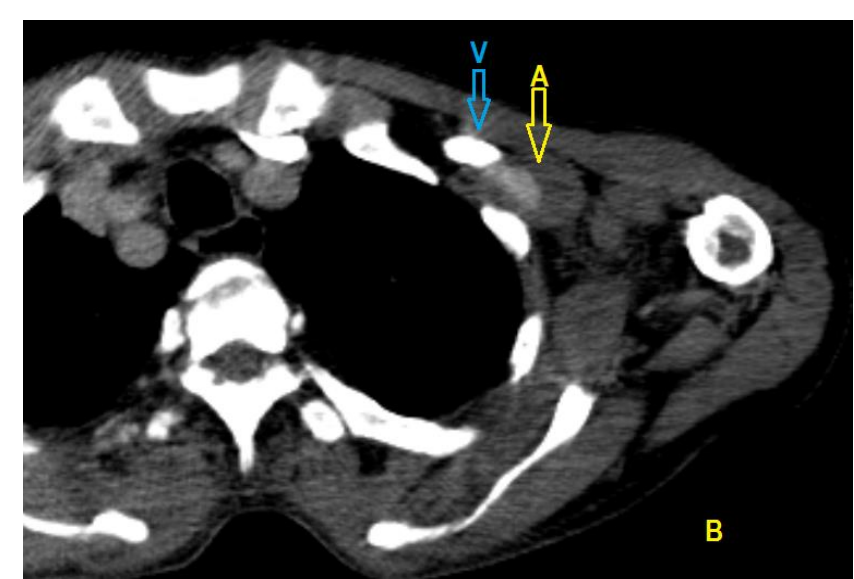

Figure 16- (Case 3): B) d-MDCTV- Axial image showing partially thrombosed axillary artery aneurysm (yellow arrow) posterior to the densely opacified axillary vein (blue arrow).

DOI: 10.21608/bmfj.2019.16833.1054 


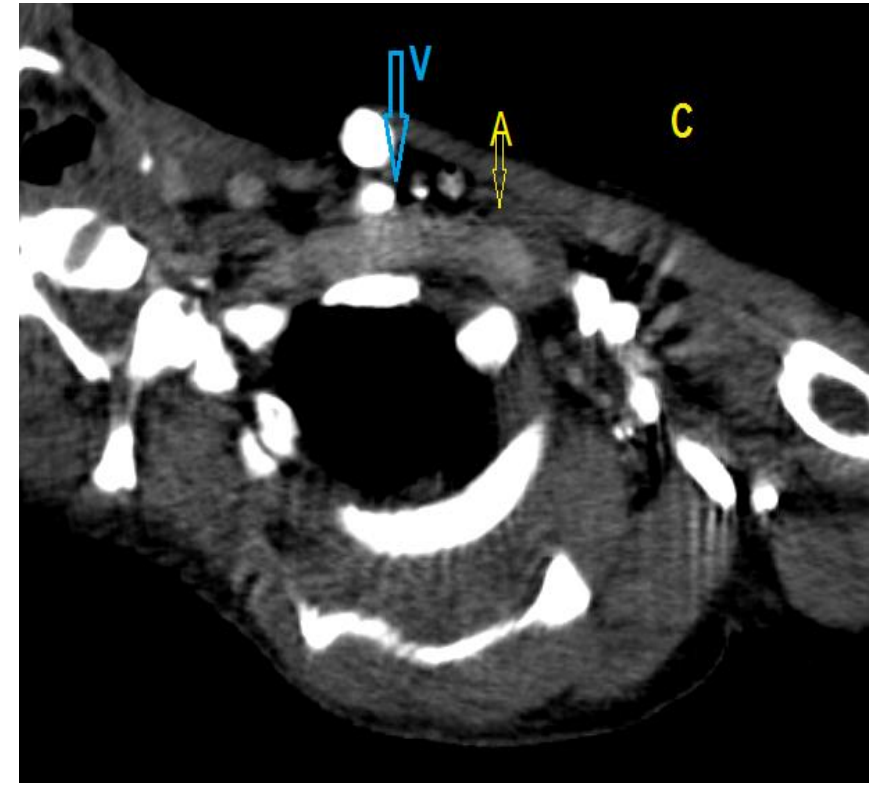

Figure 17- (Case 3): C) d-MDCTV- Axial oblique reformatted image for the axillary artery aneurysm.

\section{Discussion}

In 2018 vascular access Practice Guidelines of the ESVS, Schmidli et al (3) reported that, DUS is recommended as the first line imaging modality in suspected DVA. If CVOD cannot be reliably excluded by DUS, additional imaging methods will be necessary \& the evaluation of persistent arm edema after VA construction by fistulography or $\mathrm{CT}$ is recommended to evaluate ipsilateral central venous outflow.

\section{As regarding DUS:}

In clinical practice, the most accessible, relatively inexpensive, rapid, non-invasive and repeatable method of DVA monitoring is represented by the DUS. However, it is necessary to standardize it in order to be effective and represent a valid tool among the various specialists involved in the hemodialysis patient care (2).Therefore, in our study we followed a unified systematic protocol in DVA examination and prescription \& represent our data in illustrative diagrams for the clinicians.

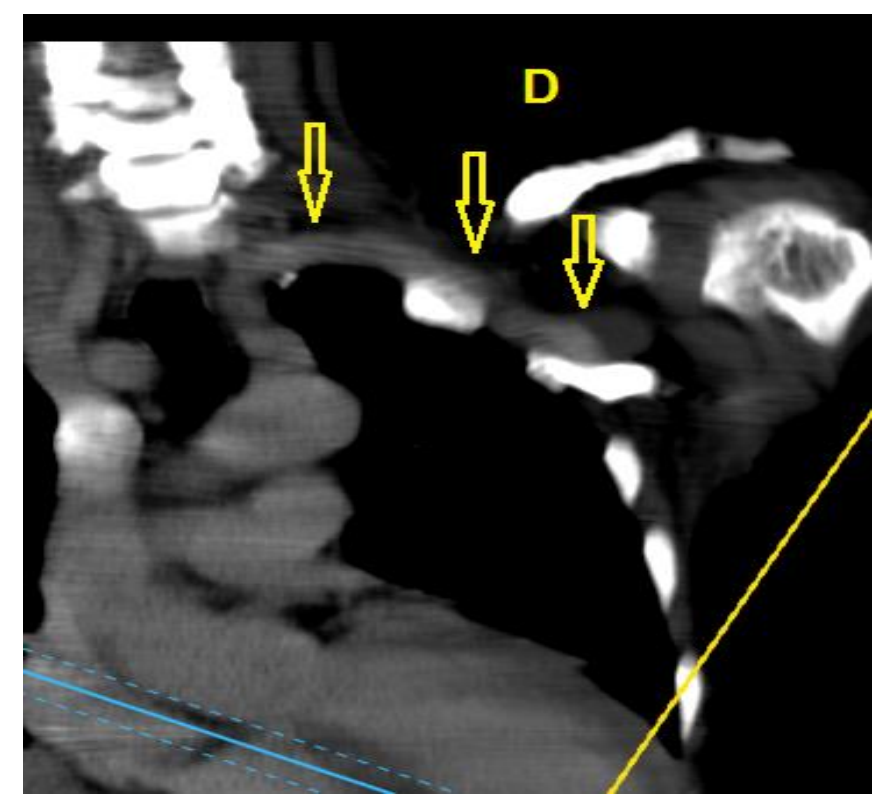

Figure 18- (Case 3): D) d-MDCTV - MIP image for the axillary artery aneurysm

Surgery performed for most of our study patients confirmed our diagnostic data (fig 8).

DUS Limitation: According to KDOQI recommendations, clinical suspicion of the diagnosis should be confirmed by either fistulography or CTA. DUS is generally less useful (3).So, the assessment of the central venous outflow is essential, as the SCV frequently shows venous stenosis in patients on dialysis (8).

We experienced that, DUS is the gold standard in the peripheral vessels anatomical \& hemodynamic evaluation. If CVODs cannot be definitely excluded, further assessment by other diagnostic modality is mandatory, because it is possible to completely change the treatment plan.

\section{As regarding direct MDCTV:}

Nan et al(4), concluded that MDCTV is the first choice to evaluate the condition of CVODs of hemodialysis patients.Direct or indirect methods can be used with injection into a superficial vein of the same limb or into the other limb, respectively. (9) Carefully 
tailored protocols CT venography allows a quick, operator-independent, relatively inexpensive, and detailed cross-sectional depiction of the venous anatomy(9).

In Nan et al indirect MDCTV study (4), 60 hemodialysis patients with swelling of upper limbs were scanned by 128-MDCT and totally 80-100 ml non-ionic CM was injected into each of the patients via the peripheral veins of the contralateral limb. The monitoring point set in the development of the lumen of inferior vena cava.

In Baz et al. study (10), dynamic CT angiography (d CTA) was done using 64 multi-detectors CT scanner in a dynamic phase. The region of interest (ROI) placed at ascending aorta. $90 \mathrm{ml}$ contrast medium is followed by $40 \mathrm{ml}$ saline was injected in the opposite arm. In Soliman et al. study (7), d CTA examination was done for 30 patients, using same protocol as the previous study (10).

In our study, we followed a unified systematic diagnostic approach for DVA assessment, and as part of this protocol, we selected certain cases for additional d-MDCTV examination. This selection was based mainly on doubt about central veins condition, in addition to clinician request when he needed further confirmation.

In our study we performed d-MDCTV examinations for 25 patients (Group B study sample), using simple 8 channels MDCT machine \& single head injector. Injection of only $50 \mathrm{ml} \mathrm{CM}$ was done which are diluted by adding $80-100 \mathrm{ml}$ saline. The diluted CM was injected in $2.5 \mathrm{ml} / \mathrm{s}$ rate into any peripheral vein in the dorsum of the hand or forearm of the limb harboring the VA.

\section{Our experienced d-MDCTV technique limitations:}

- The arterial inflow did not enhance in this study \& so the arterial inflow as well as the VA anastomosis evaluation was very limited. Nevertheless, we succeeded in demonstration a case of axillary artery aneurysm by d-MDCTV in the axial raw data cuts \& MIP images (Case 3).

- The VA flow was faintly enhanced with CM, because the CM density was continuously diluted by the non-enhanced arterial inflow and this relatively hindering VA \& venous outflow evaluation, especially if they are surrounded by multiple overlapping enhanced dilated venous collaterals. As a consequence, the reconstructed 3D images were hardly demonstrating the VA. So, demonstration of branchouts \& venous stenosis was occasionally limited.

- Direct MDCTV always requires a venous puncture in order to inject the $\mathrm{CM}$ agent in the same limb harboring DVA. This is in contrast to the doctrine of venous preservation, where punctures of peripheral veins should be kept to a minimum, as recommended by the DOQI guidelines. Nevertheless, in our study, we followed up patients during \& after d-MDCTV performance. None of our study sample individuals experienced study induced significant vascular injury.

- Radiation is an inherent risk when using CT imaging. Nevertheless, the associated risk of an increased radiation exposure is outweighed by the diagnostic benefits and subsequent changes in therapy plans for patients with suspected CVOD. 


\section{However, we also experienced that:}

- Despite of limited ability of d-MDCTV in illustration of arterial inflow, dialysis access graft (DAG) \& AVF anastomosis, its ability to demonstrate VA \& peripheral venous stenosis, aneurysms and thrombosis is feasible.

- Despite of limited ability of d-MDCTV to perform corrective intervention immediately, when a hemodynamically significant stenosis detected, compared to convention digital subtraction (DSA) studies, d-MSCTV has an advantage that it offers information regarding not only the vessel lumen but also the vessel wall and peri-vascular structures.

- Indirect MDCTV \& Dynamic CTA evaluate peripheral artery diseases much better than d-MDCTV; however, the amount of CM used in d-MDCTV is much less than that used with the indirect MDCTV \& dynamic CTA studies. Also, a CT apparatus with more limited capabilities (8-detectors) could be used in performing d-MDCTV study. In contrast, one of the minimum requirements for more advanced CTA applications, is a 64-channel CT (5).

- d-MDCTV can provide excellent visualization of the central veins. The decision of surveillance of the existing VA versus construction of a new one is far most affected by d-MDCTV results. Moreover, the site of construction a new access is also affected by its results.

\section{Conclusion:}

Following a unified, systematic DUS protocol of examination \& data prescription is the $1^{\text {st }}$ diagnostic line in DVA malfunction assessment. This method of DVA analysis allows the possibility to have a tool able to share information and competences between radiologist \& clinicians. Following this protocol, occasionally, the assessment is still incomplete, mainly as regarding to the central veins condition. We suggest that d-MDCTV, as a minimally invasive procedure; can perform this task \& complete the DVA assessment successfully, especially with limited capabilities of the available resources. Furthermore, it can provide valuable information about the other causes of DVA

\section{References:}

1. J. A. Pietryga, M. D. Little, and M. L. Robbin, "Sonography of arteriovenous fistulas and grafts," in Seminars in dialysis, vol. 30, no. 4, pp. 309318, 2017.

2. Nalesso F, Garzotto F, Petrucci I, Samoni S, Virzì GM, Gregori D, et al., Standardized Protocol for Hemodialysis Vascular Access Assessment: The Role of Ultrasound and ColorDoppler. Blood purification. 2018;45(1-3):260-269.

3. Schmidli J, Widmer MK, Basile C, de Donato G, Gallieni M, Gibbons CP, et al., Editor's choicevascular access: 2018 clinical practice guidelines of the European Society for Vascular Surgery (ESVS). European Journal of Vascular and Endovascular Surgery. 2018 Jun 1;55(6):757-818.

4. Nan LI, Chen B, Lin R, Dai H, Tang K, Wen C, et al., The use of multi-detector computed tomography venography and three dimensional reconstruction image in evaluating the central venous occlusive disease of hemodialysis patients. Chinese Journal of Nephrology. 2018 Jan 1;34(8):561-566.

5. D. J. Murphy, A. Aghayev, and M. L. Steigner, "Vascular CT and MRI: a practical guide to imaging protocols," Insights Imaging, vol. 9, no. 2, pp. 215-236, 2018.

6. Thomsen HS, Webb JA, editors. "Contrast Media: Safety Issues and ESUR Guidelines". Springer Science \& Business Media; 2014 Jan 10.

7. H. Soliman, T. Raafat, and Y. M. Abdelhamid, "Angiographic mapping of AV fistula related vascular complications in ESRD via multislice CT; adjuvant role in correlation with CDUS," Egypt. J. Radiol. Nucl. Med., vol. 46, no. 3, pp. 665-674, 2015.

8. Meyer M, Geiger N, Benck U, Rose D, Sudarski S, Ong MM, et al., Imaging of patients with complex hemodialysis arterio-venous fistulas using time- 
resolved dynamic ct angiography: comparison with duplex ultrasound. Scientific reports. 2017 Oct 2;7(1):12563.

9. Grewal S., Sutphin P., Kalva S.P. (2018) CT Venography. In: Nikolaou K., Bamberg F., Laghi A., Rubin G. (eds) Multislice CT. Medical Radiology. Springer, Cham

10. Baz AA, Naguib MM, Kamel AI, El-Khashab SO. Multi-slice CT angiography versus duplex ultrasound in detection of stenosis of access arteriovenous fistulas and grafts in dysfunctional hemodialysis. The Egyptian Journal of Radiology and Nuclear Medicine. 2016 Dec 1;47(4):1459-1466.

To cite this article: Ahmed F. Yousef, Hamada M. Khater, Hend A. AbdElrazik Role of Doppler Ultrasonography Supplemented with Direct CT Venography in Dialysis Access Assessment BMFJ, 2019: 36 (2) DOI: 10.21608/bmfj.2019.18733.1140 